\title{
Magnetism at spinel thin film interfaces probed through soft x-ray spectroscopy techniques
}

\author{
R. V. Chopdekar, ${ }^{1,2, *}$ M. Liberati, ${ }^{3}$ Y. Takamura, ${ }^{4}$ L. Fitting Kourkoutis, ${ }^{1}$ J. S. Bettinger ${ }^{2}$ B. \\ B. Nelson-Cheeseman, ${ }^{2}$ E. Arenholz, ${ }^{3}$ A. Doran, ${ }^{3}$ A. Scholl, ${ }^{3}$ D. A. Muller, ${ }^{1}$ and Y. Suzuki ${ }^{2}$ \\ ${ }^{1}$ School of Applied and Engineering Physics, Cornell University, Ithaca, NY 14853 \\ ${ }^{2}$ Department of Materials Science and Engineering, \\ University of California, Berkeley, Berkeley, CA 94720 \\ ${ }^{3}$ Advanced Light Source, Lawrence Berkeley National Laboratory, Berkeley, CA 94720 \\ ${ }^{4}$ Department of Chemical Engineering and Materials Science, \\ University of California, Davis, Davis, CA 95616
}

(Dated: December 16, 2009)

\begin{abstract}
Magnetic order and coupling at the interfaces of highly spin polarized $\mathrm{Fe}_{3} \mathrm{O}_{4}$ heterostructures have been determined by surface sensitive and element specific soft x-ray spectroscopy and spectromicroscopy techniques. At ambient temperature, the interface between paramagnetic $\mathrm{CoCr}_{2} \mathrm{O}_{4}$ or $\mathrm{MnCr}_{2} \mathrm{O}_{4}$ and ferrimagnetic $\mathrm{Fe}_{3} \mathrm{O}_{4}$ isostructural bilayers exhibits long range magnetic order of Co, $\mathrm{Mn}$ and $\mathrm{Cr}$ cations which cannot be explained in terms of the formation of interfacial $\mathrm{MnFe}_{2} \mathrm{O}_{4}$ or $\mathrm{CoFe}_{2} \mathrm{O}_{4}$. Instead, the ferrimagnetism is induced by the adjacent $\mathrm{Fe}_{3} \mathrm{O}_{4}$ layer and is the result of the stabilization of a spinel phase not achievable in bulk form. Magnetism at the interface region is observable up to $500 \mathrm{~K}$, far beyond the chromite bulk Curie temperature of 50-95 K.
\end{abstract}

PACS numbers:

\section{INTRODUCTION}

Studies of ferromagnetic interfaces have resulted in the discovery of new coupling phenomena and development of associated technological devices since Meiklejohn and Bean discovered exchange coupling between ferromagnetic $\mathrm{Co}$ and antiferromagnetic $\mathrm{CoO}$ in $1956 .{ }^{1}$ Other examples include oscillatory Rudderman-Kittel-KasuyaYoshida coupling that in metallic multilayers leads to giant magnetoresistance (GMR), ${ }^{2}$ positive exchange bias in $\mathrm{FeF}_{2}-\mathrm{Fe}$ bilayers, ${ }^{3}$ and perpendicular exchange coupling in $\mathrm{Co} / \mathrm{Pt}$ multilayers. ${ }^{4}$ Beyond metallic elements and alloy materials, complex oxide spinel structure ferrites have provided model systems for the study of phenomena such as modified superexchange interactions, ${ }^{5}$ perpendicular exchange coupling ${ }^{6}$ and nearly ideal exchange interactions. ${ }^{7}$ Margulies et al. showed that modified superexchange interactions across antiphase boundaries give rise to anomalously large saturation fields and quasi-random zero-field magnetic moment distribution. ${ }^{5}$ Ijiri et al. demonstrated perpendicular coupling of antiferromagnetic $\mathrm{CoO}$ spins with the net $\mathrm{Fe}_{3} \mathrm{O}_{4}$ moment in $\mathrm{Fe}_{3} \mathrm{O}_{4} / \mathrm{CoO}$ superlattices via neutron diffraction experiments. ${ }^{6}$ Nearly ideal exchange coupling has been observed in bilayers of hard and soft spinel ferrite layers, suggesting that in some materials systems simple theoretical models may be applied. ${ }^{7}$

Recently, isostructural spinel bilayers have been incorporated into magnetic tunnel junctions, resulting in significant improvements in junction magnetoresistance (JMR) ${ }^{8-10}$ In these junctions, magnetically harder $\mathrm{CoCr}_{2} \mathrm{O}_{4}(\mathrm{CCO})$ and $\mathrm{NiMn}_{2} \mathrm{O}_{4}$ barrier layers strongly couple to the softer $\mathrm{Fe}_{3} \mathrm{O}_{4}$ above and below their respective Curie temperatures $\left(\mathrm{T}_{c}\right)$, thus creating a hybrid magnetic tunnel junction/spin filter device. This new device's multifunctional behavior is possible due to the strong magnetic coupling at the isostructural spinelspinel interface and no magnetic coupling at the nonisostructural interface. ${ }^{10}$ Correlating the structure and the origin of the magnetism from multiple magnetic species at the oxide interfaces is crucial in explaining the large JMR and strong exchange coupling observed in $\mathrm{Fe}_{3} \mathrm{O}_{4}$ based heterostructures.

In this paper, we studied the magnetism at the isostructural spinel interfaces of ferrimagnetic $\mathrm{Fe}_{3} \mathrm{O}_{4}$ and paramagnetic insulators $\mathrm{CCO}$ and $\mathrm{MnCr}_{2} \mathrm{O}_{4}(\mathrm{MCO})$ in order to understand the origin of the strong exchange coupling between the two layers. Surprisingly, we observed induced ferrimagnetism in the paramagnetic insulator layers by the adjacent ferrimagnet up to 500 $\mathrm{K}$. Nanoscale roughness and the formation of interfacial $\mathrm{MnFe}_{2} \mathrm{O}_{4}$ or $\mathrm{CoFe}_{2} \mathrm{O}_{4}$ cannot sufficiently explain the high-temperature magnetic behavior of $\mathrm{Co}, \mathrm{Mn}$ and $\mathrm{Cr}$ in the ferrimagnet-paramagnet bilayers. We conclude that the magnetization induced in the paramagnetic layer is due to a proximity effect to the isostructural ferrimagnet, and the stabilization of a spinel phase at the interface not attainable in bulk form.

\section{EXPERIMENTAL}

We have synthesized epitaxial thin films and multilayers of $\mathrm{Fe}_{3} \mathrm{O}_{4}, \mathrm{CCO}$ and $\mathrm{MCO}$ grown by pulsed laser deposition (PLD) on (110) oriented $\mathrm{SrTiO}_{3}$ (STO) and Nb-doped STO substrates. Four sets of samples with uniform thickness were prepared: $20 \mathrm{~nm}$ single layers of $\mathrm{Fe}_{3} \mathrm{O}_{4}$ (hereafter referred to as SF samples), $40 \mathrm{~nm}$ single layers of $\mathrm{CCO}$ or MCO (SC or SM samples), $20 \mathrm{~nm}$ $\mathrm{Fe}_{3} \mathrm{O}_{4}$ with varying thickness of $\mathrm{CCO}$ or MCO cap layer 
(SFC or SFM samples), and $40 \mathrm{~nm}$ CCO or MCO layers with a $5 \mathrm{~nm} \mathrm{Fe}{ }_{3} \mathrm{O}_{4}$ cap layer (SCF or SMF samples).

Commercial sintered powder targets of stoichiometric single-phase spinel oxides were used for ablation at an energy density of $1-1.5 \mathrm{~J} / \mathrm{cm}^{2}$. Deposition parameters for the single layers are as follows: $\mathrm{Fe}_{3} \mathrm{O}_{4}$ in a vacuum of better than $4 \times 10^{-6}$ Torr at a substrate temperature of $450{ }^{\circ} \mathrm{C}$, and $\mathrm{MCO}$ and $\mathrm{CCO}$ in 25 mTorr of $\mathrm{O}_{2}$ at 600 ${ }^{\circ} \mathrm{C}$. In order to prevent $\mathrm{Fe}_{3} \mathrm{O}_{4}$ from oxidizing during the growth of subsequent layers in an oxygen ambient, the $\mathrm{Fe}_{3} \mathrm{O}_{4}$ layer deposition was followed by 1 monolayer of chromite deposited in vacuum and then the remainder of the chromite layer grown in 25 mTorr of a $1 \% \mathrm{O}_{2} / 99 \%$ $\mathrm{N}_{2}$ mixture at $450{ }^{\circ} \mathrm{C}$. Additional spectra were taken on a single crystal sample of $\mathrm{CoFe}_{2} \mathrm{O}_{4}$ (CFO).

Structural and chemical characterization of the thin films included atomic force microscopy to characterize the surface morphology of the deposited films, 2 and 4circle x-ray diffraction to study the crystallinity of the epitaxial spinel layers deposited on perovskite substrates, and Rutherford Backscattering Spectrometry (RBS) to assess film thickness and composition. Further studies of the oxide interfaces were performed via scanning transmission electron microscopy (STEM) and electron energy loss spectroscopy (EELS).

Temperature and field-dependent magnetization measurements for both single layers and bilayers were performed in a Quantum Design MPMS 5XL magnetometer with field oriented along the [001] in-plane direction for (110) oriented films. In order to determine the details of the interface magnetism, element specific and interface sensitive probes of X-ray absorption spectroscopy (XAS), X-ray magnetic circular dichroism (XMCD) and photoemission electron microscopy (PEEM) were used. Soft x-ray absorption spectroscopy experiments in total electron yield mode were performed from $10 \mathrm{~K}$ to $300 \mathrm{~K}$ in fields of up to $0.8 \mathrm{~T}$ at beamlines 4.0.2 ${ }^{11}$ and $6.3 .1^{12}$ of the Advanced Light Source (ALS) at Lawrence Berkeley National Laboratory. Spectro-microscopy measurements took place at the PEEM2 microscope (beamline 7.3.1.1) $)^{13}$ of the ALS at $300 \mathrm{~K}$ to $550 \mathrm{~K}$ in zero magnetic field. The incident $\mathrm{x}$-ray propagation vector was projected onto the [001] or [110] in-plane crystallographic direction of the sample.

\section{RESULTS AND DISCUSSION}

\section{A. Structural and Chemical Characterization}

Surface morphology for spinel single films and bilayers on (110) oriented STO substrates was smooth, with maximum RMS surface roughness of $0.9 \mathrm{~nm}$, which is of the order of the spinel unit cell. Four circle X-ray diffraction analysis indicated that each component layer was single phase and epitaxially matched to the underlying (110) oriented substrates. While RBS analysis confirmed the stoichiometry of the $40 \mathrm{~nm}$ single layers, the composition

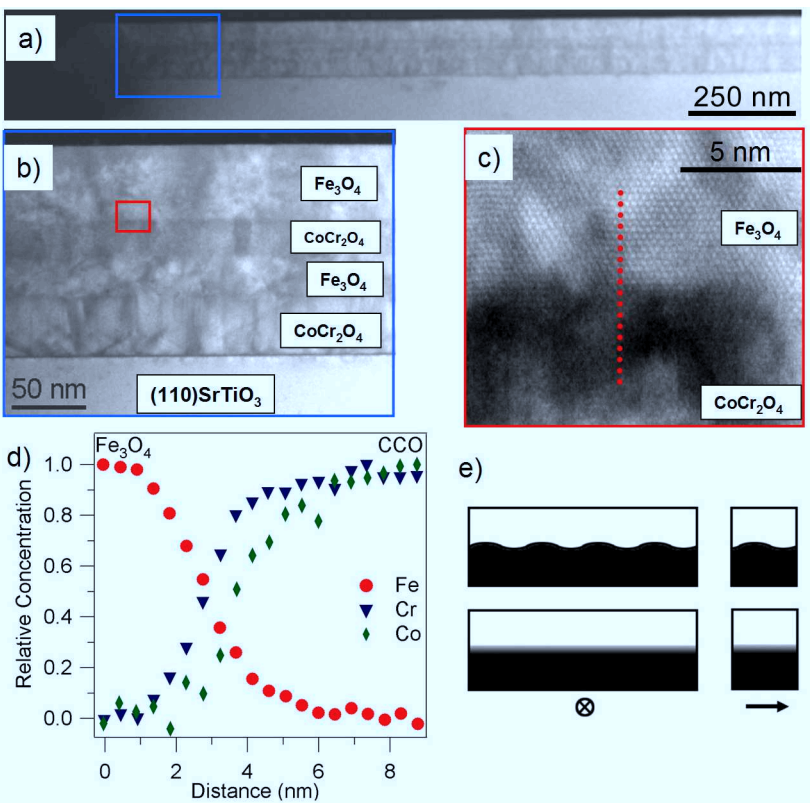

FIG. 1: (Color online) High-angle annular dark field STEM images of a SCFCF multilayer: (a) low magnification, (b) mid magnification, (c) high magnification, and (d) integrated EELS intensity for $\mathrm{Fe}, \mathrm{Cr}$, and Co edges across the dotted line indicated in c). Two cases of interface roughening are shown in (e), with the top schematic indicating a chemically distinct but structurally rough interface and the bottom schematic indicating a chemically interdiffused interface. The arrow indicates the direction of the incident electron beam.

of $10 \mathrm{~nm}$ and thinner layers and any interdiffusion in such layers could not be quantified via RBS alone.

STEM analysis of $\mathrm{Fe}_{3} \mathrm{O}_{4} / \mathrm{CCO}$ based heterostructures in a previous study indicated that the spinel interfaces were chemically distinct to within the $2 \mathrm{~nm}$ probe size, ${ }^{14}$ but interface roughness could induce magnetic coupling between electrode layers across a thin insulating barrier layer due to so-called orange-peel coupling. ${ }^{15}$ In order to assess the possible role of roughness at these oxide interfaces, cross-section samples of multilayers were analyzed with STEM. Figures 1(a)-(d) show micrographs and EELS linescans at the upper $\mathrm{CCO} / \mathrm{Fe}_{3} \mathrm{O}_{4}$ interface for a (110) oriented STO // $41 \mathrm{~nm} \mathrm{CCO} \mathrm{//} 26 \mathrm{~nm} \mathrm{Fe}_{3} \mathrm{O}_{4}$ // $24 \mathrm{~nm} \mathrm{CCO} \mathrm{//} 43 \mathrm{~nm} \mathrm{Fe} \mathrm{O}_{4}$ (SCFCF) sample. While defects such as low-angle grain boundaries occur in the spinel layers due to the large $(-7 \%)$ lattice mismatch between the spinel unit cell and the STO substrate, the film is crystalline with all grains matched to the (110) out of plane orientation of the substrate. Examination of the $\mathrm{Fe}_{3} \mathrm{O}_{4}$ - $\mathrm{CCO}$ interface shows distinct layers in the low magnification high-angle annular dark field STEM images (Fig. 1(a)), but chemical mapping of the interface (Fig. 1(d)) shows a lack of sharp chemical transition between the layers, and the transition width is of order $2 \mathrm{~nm}$ on both sides of the nominal interface.

The transition at the spinel interface maybe be explained in terms of roughness of the initial layer (Fig. 


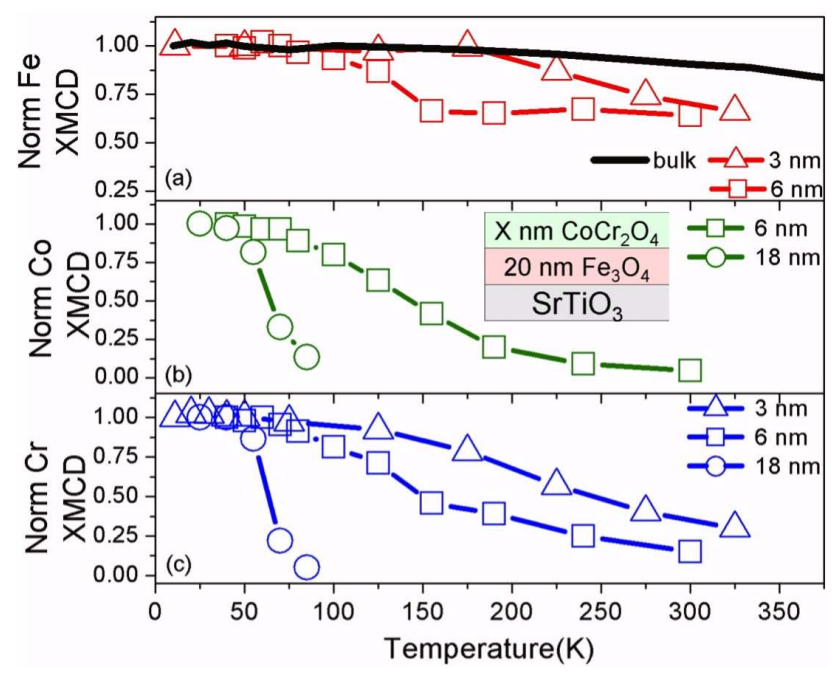

FIG. 2: (Color online) Temperature dependence of the saturation magnetization for SFC samples as a function of CCO layer thickness as measured by a SQUID magnetometer (solid line, $3 \mathrm{~nm}$ CCO sample) and saturation asymmetry of XMCD signal measured on (a) Fe, (b) $\mathrm{Co}$, and (c) $\mathrm{Cr} \mathrm{L}_{2,3}$ edges normalized to $12 \mathrm{~K}$ values.

1(e) top) or the high kinetic energy of the subsequent layer's deposition inducing an interdiffusion at the interface (Fig. 1(e) bottom). The offset in the $\mathrm{Cr}$ and $\mathrm{Co}$ concentration profiles (Fig. 1(d)) can not be explained by interface roughness alone, and suggests that cation interdiffusion is present at these interfaces. ${ }^{16}$ It is likely that the sample has contributions from both roughness and interdiffusion as the large epitaxial misfit strain induced defects such as the low angle grain boundaries seen in the STEM micrographs, and the highly energetic PLD plume may accelerate diffusion of the layers. ${ }^{17}$ As the deposition rate of the $43 \mathrm{~nm}$ top $\mathrm{Fe}_{3} \mathrm{O}_{4}$ layer was slower than that of the CCO or MCO layers under study, the STEM sample was held at $450{ }^{\circ} \mathrm{C}$ for a longer duration than the bilayers examined with XAS and XMCD. Thus, $4 \mathrm{~nm}$ represents an upper bound for the size of the intermixed region if we assume that the EELS elemental concentration transition width is wholly due to interdiffusion during the PLD growth process.

\section{B. Magnetic Characterization}

In order to determine the nature of magnetic interactions at the $\mathrm{Fe}_{3} \mathrm{O}_{4} /$ chromite interfaces and the origin of the induced ferrimagnetism in the paramagnetic chromite spinels, we measured the X-ray absorption cross section as function of magnetic field, polarization and temperature by measuring the sample drain current in total electron yield mode with a probing depth of approximately $5 \mathrm{~nm}$ for $\mathrm{Fe}_{3} \mathrm{O}_{4} \cdot{ }^{18}$ Figures 2(a)-(c) show the temperature dependence of the dichroism at the $\mathrm{Fe}, \mathrm{Co}$ and $\mathrm{Cr}$ edges normalized to the value at $12 \mathrm{~K}$, as well as the bulk
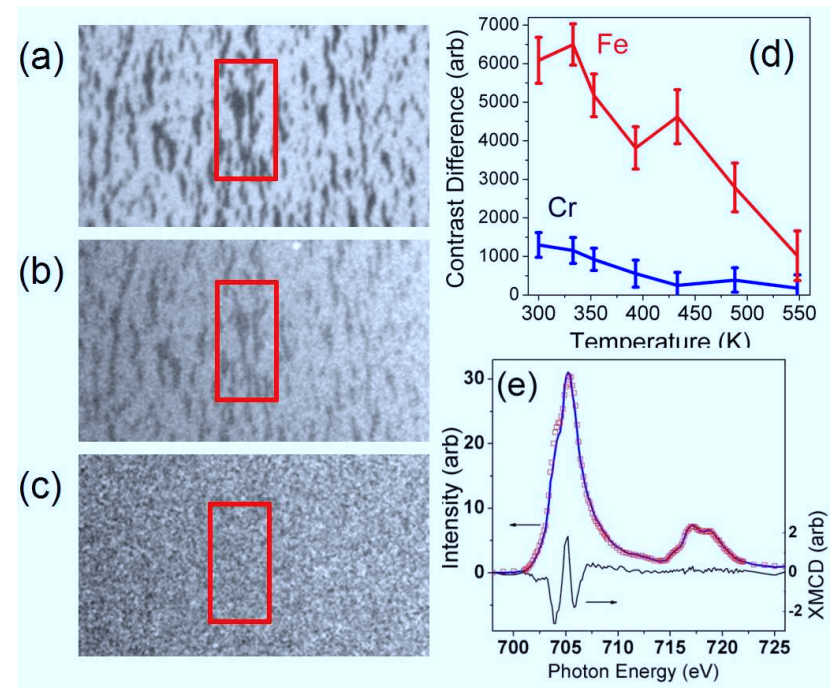

FIG. 3: (Color online) PEEM domain images from the 20 $\mathrm{nm} / 3 \mathrm{~nm}$ SFC sample. The $5 \mu \mathrm{m} \times 2.8 \mu \mathrm{m}$ boxed region highlights identical domain structure with same polarity on the $\mathrm{Fe}(\mathrm{a})$ and $\mathrm{Cr}$ (b) edges, but no detectable polarization of Co (c). Contrast difference for Fe and $\mathrm{Cr}$ edges (d) show that ferromagnetism persists up to $500 \mathrm{~K}$. Room temperature Fe spectroscopy (e) on dark domains (solid line) compared to light domains (open squares) with resulting XMCD difference.

magnetization as measured by superconducting quantum interference device (SQUID) magnetometry for a $20 \mathrm{~nm}$ / $3 \mathrm{~nm}$ SFC bilayer. While the $\mathrm{Cr}$ and Co XMCD signals sample the average of the entire $3 \mathrm{~nm}$ CCO layer in the $20 \mathrm{~nm} / 3 \mathrm{~nm}$ SFC sample, the Fe XMCD only samples a few nm of the $\mathrm{Fe}_{3} \mathrm{O}_{4}$ closest to the interface is measured.

Element-specific coercive fields were extracted from XMCD hysteresis measurements, with the $\mathrm{Cr}$ and Fe edge coercive fields matching as a function of temperature. However, the magnitude of the $\mathrm{Cr}$ dichroism decreases more quickly with increasing temperature than the $\mathrm{Fe}$ edge dichroism. The normalized Fe and Cr XMCD signals for the $20 \mathrm{~nm} / 3 \mathrm{~nm}$ SFC sample fell sharply as a function of increasing temperature above $175 \mathrm{~K}$ (Figures $2(\mathrm{a})$ and (c)) when compared to the magnetization of the entire sample as measured by SQUID magnetometry (Figure 2(a), solid line).

Similar XMCD measurements performed on a $20 \mathrm{~nm} /$ $18 \mathrm{~nm}$ SFC bilayer probe the $\mathrm{Cr}$ and Co moments away from the $\mathrm{Fe}_{3} \mathrm{O}_{4}$ interface and showed only bulk-like magnetism that disappears at approximately $80 \mathrm{~K}$ (Figure $2(\mathrm{~b})$ and (c)). With a single layer CCO film deposited on $\mathrm{Nb}: \mathrm{STO}$, we observed bulk-like magnetic properties with a $\mathrm{T}_{c}$ of $95 \mathrm{~K}$, a coercive field of $2 \mathrm{~T}$ and a saturation magnetization of $0.23 \mu_{B}$ /formula unit at $5 \mathrm{~K}$. A similar layer of MCO had a $\mathrm{T}_{c}$ of $50 \mathrm{~K}$, a coercive field of $0.1 \mathrm{~T}$ and a saturation magnetization of $0.93 \mu_{B} /$ formula unit at $5 \mathrm{~K}$. Magnetic characterization of powder taken from the CCO and MCO PLD targets match these Curie temperature values as well as those found in literature. ${ }^{19}$ Thus while the single layer CCO has bulk-like character- 


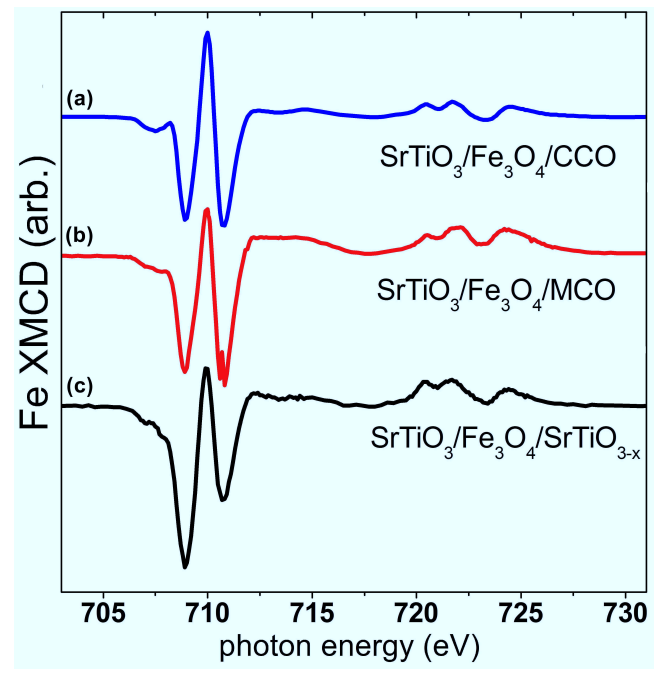

FIG. 4: (Color online) Room temperature Fe $\mathrm{L}_{2,3} \mathrm{XMCD}$ spectra for $\mathrm{Fe}_{3} \mathrm{O}_{4}$ bilayers on (110) oriented STO: (a) CCO cap layer, (b) MCO cap layer, and (c) $\mathrm{SrTiO}_{3-x}$ cap layer.

istics, in the vicinity of the interface with $\mathrm{Fe}_{3} \mathrm{O}_{4}$ the $\mathrm{Cr}$ and Co magnetization drastically differs from bulk. If we assumed no influence of the $\mathrm{Fe}_{3} \mathrm{O}_{4}$ on $\mathrm{CCO}$ above $\mathrm{T}_{c}^{C C O}$, the persistence of $\mathrm{Cr}$ moment above room temperature could not be explained.

Magnetic domain images were taken of uncapped and chromite capped $\mathrm{Fe}_{3} \mathrm{O}_{4}$ layers via PEEM. $\mathrm{Fe}_{3} \mathrm{O}_{4}$ domains coupled to $\mathrm{Cr}$ in $\mathrm{CCO}$ capping layers of thickness $3 \mathrm{~nm}$ as shown in Figure 3(a) and (b) as well as $6 \mathrm{~nm}$. The domain structure of the octahedral $\mathrm{Cr}$ moments is coupled ferromagnetically to the octahedral $\mathrm{Fe}$ moments in $\mathrm{Fe}_{3} \mathrm{O}_{4}$ as deduced from the domain contrast observed in PEEM. Domain structure could not be observed on the Co $\mathrm{L}_{3}$ edge even at $300 \mathrm{~K}$ (Figure $3(\mathrm{c})$ ) to within the experimental resolution of the microscope, but persisted up to $500 \mathrm{~K}$ on the $\mathrm{Cr} \mathrm{L}_{3}$ edge. The difference in contrast for light and dark areas in Figures 3(a) and (b) is plotted as a function of temperature in Figure 3(d). The domain structure at elevated temperatures remained identical to the room temperature domain structure for both $\mathrm{Cr}$ and $\mathrm{Fe} \mathrm{L}_{3}$ edges. The contrast between light and dark domains fell to below the noise level of the measurement by $540 \mathrm{~K}$.

The enhanced long range order observed in chromite layers on $\mathrm{Fe}_{3} \mathrm{O}_{4}$ is unexpected. Its origin may be explained in terms of a high $\mathrm{T}_{c} \mathrm{CoFe}_{2} \mathrm{O}_{4}$ or $\mathrm{MnFe}_{2} \mathrm{O}_{4}$ like phase at the interface, a ferromagnetic proximity effect between the $\mathrm{Fe}_{3} \mathrm{O}_{4}$ and chromite layer, or a metastable phase at the interface not found in bulk. A localized energy scan along the $\mathrm{Fe}_{2,3}$ edges in the field of view of Figure 3(a) for both light and dark domains yields spectra (Figure 3(e)), whose difference may be compared to other $\mathrm{Fe}_{3} \mathrm{O}_{4}$ spectra to ascertain the local Fe environment. The resulting difference was similar in lineshape to data from thin film $\mathrm{Fe}_{3} \mathrm{O}_{4}$ data, ${ }^{20}$ suggesting that the $\mathrm{Fe}$ environment at the interface did not differ substan- tially from $\mathrm{Fe}$ in bulk $\mathrm{Fe}_{3} \mathrm{O}_{4}$. In order to understand the induced ferrimagnetism at the interface of these layers, we examined XAS and XMCD of the $\mathrm{Fe}, \mathrm{Cr}, \mathrm{Co}$, and $\mathrm{Mn}$ $\mathrm{L}_{2,3}$ edges in a systematic set of samples.

Figure 4 compares the $\mathrm{Fe} \mathrm{L}_{2,3}$ XMCD spectra for $\mathrm{Fe}_{3} \mathrm{O}_{4}$ with various spinel capping layers and a STOcapped sample as reference. Multiplet calculations have been compared to experimental dichroism spectra from $\mathrm{Fe}$ in various spinels ${ }^{21}$ as well as PLD-deposited $\mathrm{Fe}_{3} \mathrm{O}_{4}$ epitaxial thin films. ${ }^{22}$ The dominant contribution of each XMCD peak in Figure 4 can be approximately assigned as follows: $708.9 \mathrm{eV}$ as octahedral $\mathrm{Fe}^{2+}, 709.9 \mathrm{eV}$ as tetrahedral $\mathrm{Fe}^{3+}$, and $710.8 \mathrm{eV}$ as octahedral $\mathrm{Fe}^{3+}$. The presence of $\mathrm{Mn}$ reduces the tetrahedral $\mathrm{Fe}^{3+} 709.9 \mathrm{eV}$ peak in Figure 4 (b) when compared to similar CCO samples, which indicates that $\mathrm{Mn}$ is displacing the interfacial tetrahedral $\mathrm{Fe}^{3+}$ in a similar manner to more bulk-like manganese ferrites. To show the sensitivity of the XMCD spectrum to the average oxidation state of the interface Fe ions, we also show the Fe XMCD spectrum from a $\mathrm{Fe}_{3} \mathrm{O}_{4} / \mathrm{SrTiO}_{3-x}$ bilayer in Figure 4 (c). The $3 \mathrm{~nm} \mathrm{SrTiO}_{3-x}$ cap layer was deposited in high vacuum to prevent oxidation of the $\mathrm{Fe}_{3} \mathrm{O}_{4}$ underlayer, and the oxygen-deficient cap acts to reduce interfacial Fe ions as seen from the peak corresponding to octahedral $\mathrm{Fe}^{2+}$ increasing in intensity at the expense of the $\mathrm{Fe}^{3+}$ peaks.

The Cr, Co and Mn XAS lineshapes for single chromite layers showed almost identical structure to those of $\mathrm{Fe}_{3} \mathrm{O}_{4} /$ chromite layers (Figures 5-7 left panels). The $\mathrm{Cr}$ single and bilayer XAS lineshapes in Figure 5 were almost identical to reference Cr XAS scans of the single-phase powder CCO and MCO targets used to deposit the films. This confirms that $\mathrm{Cr}$ is found in octahedral sites as $\mathrm{Cr}^{3+}$ in both single and bilayers as compared to the expected cation distribution found in bulk chromites. ${ }^{23}$ The chemical environment deduced from the XAS of the Co and $\mathrm{Mn}$ in the bilayer is similar to that of a single chromite layer or the PLD target material, i.e., both Co and Mn are predominantly in the tetrahedral sites. However, Cr, $\mathrm{Mn}$, and Co in the bilayers showed marked increase in $\mathrm{XMCD}$ signal as compared to single layer films. The dichroism signal persisted up to room temperature for all bilayers regardless of whether or not the $\mathrm{Fe}_{3} \mathrm{O}_{4}$ layer was the cap layer or the underlayer (Figures 5-7 right panels).

The room temperature XMCD lineshape for the Co edge of the SCF sample (Fig. 6(b) spectrum 2) shows lineshape similarities to that of a $\mathrm{CFO}$ crystal which is composed of exclusively octahedral $(\mathrm{Oh}) \mathrm{Co}^{2+}$ (Fig. 6(b), spectrum 4). CCO has been determined to be a normal spinel,,$^{23}$ and contains only tetrahedral (Td) $\mathrm{Co}^{2+}$ given the strong preference of the $\mathrm{Cr}$ for octahedral sites in spinels as compared to Co. ${ }^{24,25} \mathrm{~A}$ comparison between spectra 2 (SCF sample) and 4 (CFO crystal) suggest that the room temperature XMCD for the $\mathrm{CCO}$ bilayer is a superposition of an octahedral $\mathrm{Co}^{2+}$ contribution from the interface region and a much smaller signal from tetrahedral $\mathrm{Co}^{2+}$ in bulk-like CCO. Based on the tetrahedral- 
TABLE I: Co sample XMCD spectra decomposition into contributions of magnetic tetrahedral and octahedral ions.

\begin{tabular}{ccccc}
\hline \hline Sample & Description & Top layer thickness & Temperature & Percent Co Td \\
\hline $\mathrm{CFO}$ & $\mathrm{CoFe}_{2} \mathrm{O}_{4}$ bulk crystal & - & $298 \mathrm{~K}$ & taken as $0 \%$ \\
$\mathrm{SC}$ & $(110) \mathrm{SrTiO}_{3} / \mathrm{CoCr}_{2} \mathrm{O}_{4}$ & $40 \mathrm{~nm}$ & $30 \mathrm{~K}$ & taken as $100 \%$ \\
$\mathrm{SFC}$ & $(110) \mathrm{SrTiO}_{3} / \mathrm{Fe}_{3} \mathrm{O}_{4} / \mathrm{CoCr}_{2} \mathrm{O}_{4}$ & $3 \mathrm{~nm}$ & $14 \mathrm{~K}$ & $54 \pm 1 \%$ \\
$\mathrm{SFC}$ & $(110) \mathrm{SrTiO}_{3} / \mathrm{Fe}_{3} \mathrm{O}_{4} / \mathrm{CoCr}_{2} \mathrm{O}_{4}$ & $6 \mathrm{~nm}$ & $20 \mathrm{~K}$ & $56 \pm 2 \%$ \\
$\mathrm{SFC}$ & $(110) \mathrm{SrTiO}_{3} / \mathrm{Fe}_{3} \mathrm{O}_{4} / \mathrm{CoCr}_{2} \mathrm{O}_{4}$ & $12 \mathrm{~nm}$ & $14 \mathrm{~K}$ & $92 \pm 4 \%$ \\
$\mathrm{SCF}$ & $(110) \mathrm{SrTiO}_{3} / \mathrm{CoCr}_{2} \mathrm{O}_{4} / \mathrm{Fe}_{3} \mathrm{O}_{4}$ & $5 \mathrm{~nm}$ & $298 \mathrm{~K}$ & $8 \pm 2 \%$ \\
\hline \hline
\end{tabular}
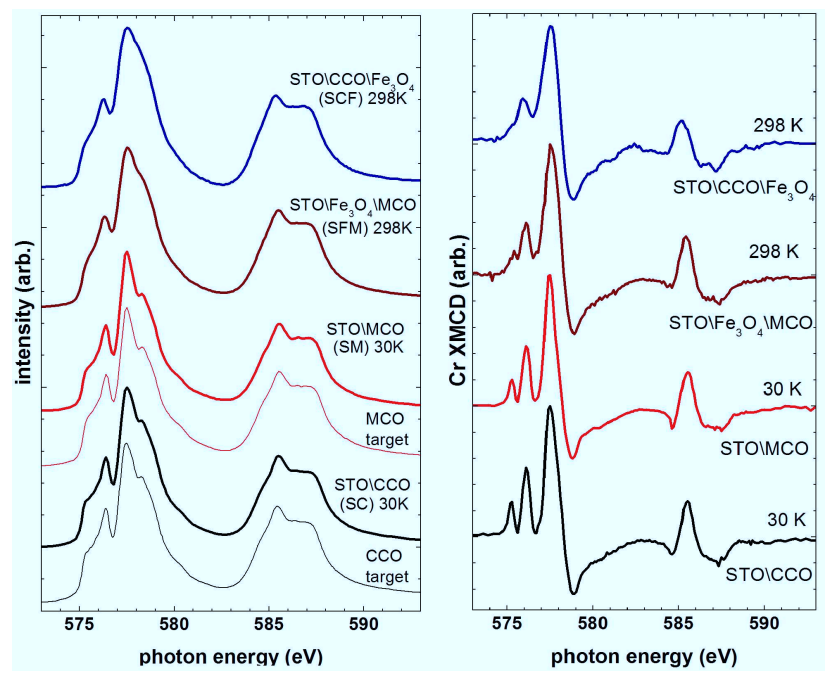

FIG. 5: (Color online) $\mathrm{Cr} \mathrm{L}_{2,3}$ (a) XAS and (b) XMCD spectra for SCF and SFM samples compared to reference $\mathrm{Cr}_{\mathrm{Oh}}^{3+}$ sample spectra.
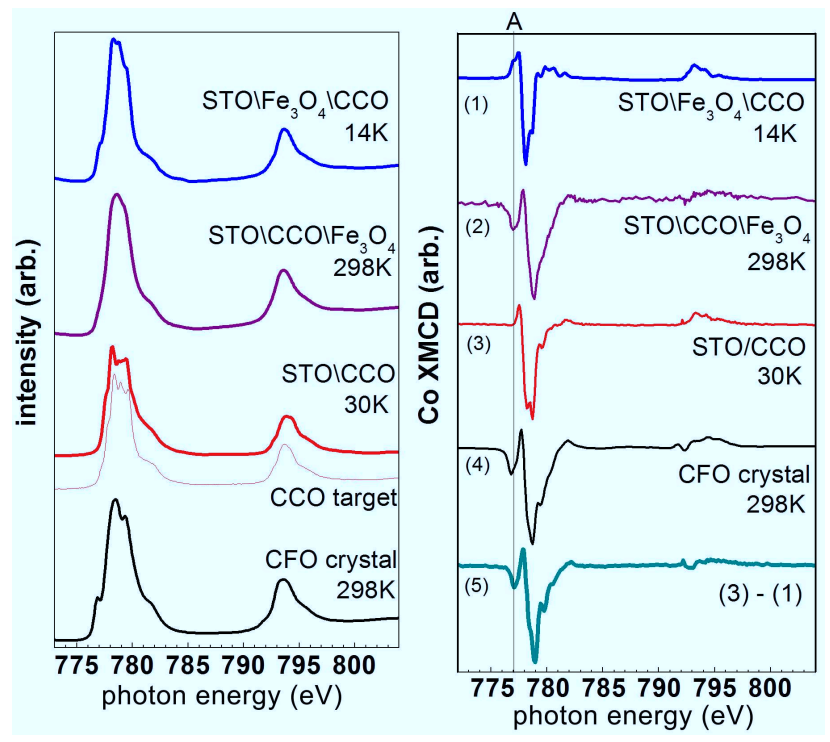

FIG. 6: (Color online) Co $\mathrm{L}_{2,3}$ (a) XAS and (b) XMCD spectra for SFC and SCF samples compared to reference $\mathrm{Co}_{T d}^{2+}$ (SC and CCO target) and $\mathrm{Co}_{\mathrm{O} h}^{2+}$ (CFO) sample spectra. Spectrum 5 is the difference between the SFC spectrum and the annealed CCO spectrum. only $\mathrm{Co}^{2+}$ lineshape for a single layer of $\mathrm{CCO}$ and the Oh-only $\mathrm{Co}^{2+}$ lineshape of a CFO crystal, we performed a least-squares fitting of the bilayer spectrum after alignment of the XMCD spectra pre-peak feature to the line indicated as 'A'. The integrals of the base $\mathrm{Td}$ and $\mathrm{Oh}$ spectra in Fig. 6 were normalized to unity, then the base spectra were used to decompose the SFC and SCF spectra to evaluate the relative contributions of $\mathrm{Co}_{O h}^{2+}$ and $\mathrm{Co}_{T d}^{2+}$.

In the SCF samples, the thickness of the $\mathrm{Fe}_{3} \mathrm{O}_{4}$ is such that only the $\mathrm{Co}$ at the $\mathrm{Fe}_{3} \mathrm{O}_{4}$ interface contributes to the XMCD measurements in total electron yield mode. In the SFC samples, Co from the entire $\mathrm{CCO}$ film is sampled including the $\mathrm{Co}$ at the $\mathrm{Fe}_{3} \mathrm{O}_{4}$ interface. The percentage of magnetic $\mathrm{Td} \mathrm{Co}$ in the room temperature bilayer spectrum (Fig. 6(b) spectrum 2) is $8 \%$ and thus the interfacial magnetic $\mathrm{Co}$ is dominated by $\mathrm{Co}_{O h}^{2+}$. For comparison, the low temperature spectrum of the SFC sample (Fig. 6(b) spectrum 1), which is a mixture of interfacial Co and whole-film CCO, is $54 \% \mathrm{Td}$. In addition, the fitted Co XMCD tetrahedral and octahedral signals in both SFC and SCF samples are antiparallel and thus are in opposing sublattices in the spinel structure.

The room temperature XMCD for the CCO bilayer is a superposition of an octahedral $\mathrm{Co}^{2+}$ contribution from the interface region and a much smaller signal from tetrahedral $\mathrm{Co}^{2+}$ in $\mathrm{CCO}$, whereas that tetrahedral $\mathrm{Co}^{2+}$ contributes more strongly to the total dichroism signal at low temperatures. This shift in Co XMCD contribution demonstrates that it is $\mathrm{Co}$ from the intermixed region at the $\mathrm{Fe}_{3} \mathrm{O}_{4} / \mathrm{CCO}$ interface that is partly responsible for the high temperature magnetism. Additionally, the strong tetrahedral component of the Co dichroism at low temperatures for the $20 \mathrm{~nm} / 3 \mathrm{~nm}$ SFC bilayer as compared to the room temperature measurement confirms that even a $3 \mathrm{~nm}$ CCO layer is distinct and has a bulklike inversion parameter, but this layer is partially magnetized by the interfacial material even above $\mathrm{T}_{c}^{C C O}$.

In addition to the octahedral Co XMCD signal from the interface, the octahedral Cr XMCD signal is strong as well, thus suggesting that the origin of the high temperature magnetism is not simply the formation of a CFO-like phase. Mohan et al. found that in cobalt ferromchromites of intermediate $\mathrm{Cr}$ concentrations, Co occupies both tetrahedral and octahedral sites and the authors assigned cation distribution as $\left(\mathrm{Fe}_{0.3}^{+3} \mathrm{Co}_{0.7}^{2+}\right)^{A}\left[\mathrm{Co}_{0.3}^{2+} \mathrm{Cr}_{0.6+y}^{3+} \mathrm{Fe}_{1.1-y}^{3+}\right]^{B} \mathrm{O}_{4}$ for $0 \leq y \leq 1 .^{26}$ 

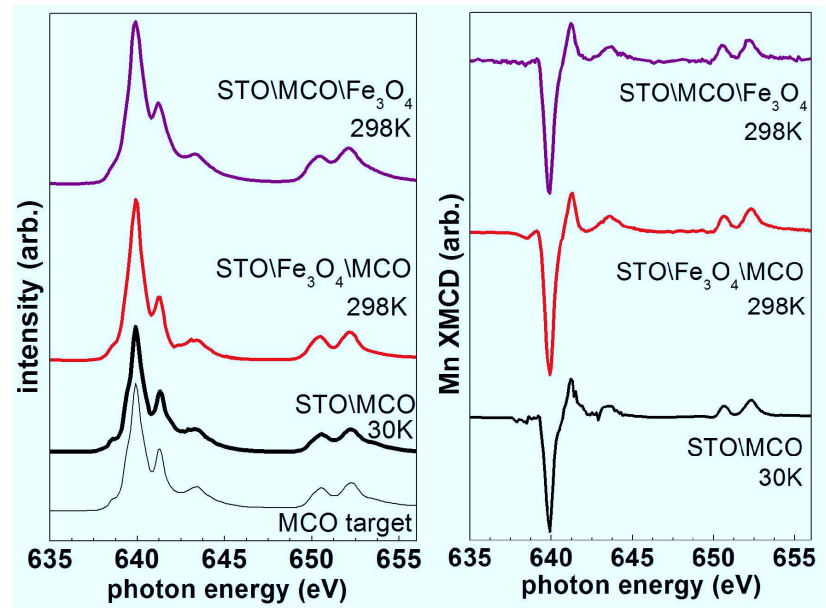

FIG. 7: (Color online) $\mathrm{Mn} \mathrm{L}_{2,3}$ (a) XAS and (b) XMCD spectra for SFM and SMF interfaces compared to reference $\mathrm{Mn}_{T d}^{2+}$ (SM and MCO target) sample spectra.

In powder samples, Cr-rich ferrites have $\mathrm{T}_{c} \mathrm{~s}$ below room temperature, with $\mathrm{T}_{c} \approx 150 \mathrm{~K}$ for $\mathrm{Fe}_{1.5} \mathrm{Cr}_{1.5} \mathrm{O}_{4} \cdot{ }^{27} \mathrm{Com}-$ bined with the cross sectional characterization of the $\mathrm{CCO} / \mathrm{Fe}_{3} \mathrm{O}_{4}$ interface and the PEEM domain contrast data, we can deduce that octahedral $\mathrm{Cr}^{3+}$ is strongly polarized by the $\mathrm{Fe}^{3+}$ in $\mathrm{Fe}_{3} \mathrm{O}_{4}$ and $\mathrm{Co}^{2+}$ at the interface is polarized to a smaller degree.

We performed a similar analysis on the MCO$\mathrm{Fe}_{3} \mathrm{O}_{4}$ interface. The XMCD lineshapes for the $\mathrm{Mn}$ in MCO and the SFM and SMF bilayers overlap to within $3 \%$ error, therefore tetrahedral $\mathrm{Mn}^{2+}$ exists exclusively at the interface. $\mathrm{Mn}^{2+}$ is expected to be in tetrahedral sites in both $\mathrm{MCO}$ and in $(\mathrm{Mn}, \mathrm{Cr}, \mathrm{Fe})_{3} \mathrm{O}_{4}$. Saksonov and Somenkov assigned $\mathrm{Mn}$ to be primarily divalent and tetrahedral in manganese-iron chromite $\left(\mathrm{Mn}_{\epsilon}^{2+} \mathrm{Fe}_{1-\epsilon}^{3+}\right)^{A}\left[\mathrm{Mn}_{1-\epsilon}^{3+} \mathrm{Fe}_{1-\epsilon}^{2+} \mathrm{Fe}_{2 \epsilon-t}^{3+} \mathrm{Cr}_{t}^{3+}\right]^{B} \mathrm{O}_{4}$ with $0.84 \leq \epsilon \leq 0.98$ and $0.5 \leq t \leq 1.5 .^{28}$ Thus, any presence of $\mathrm{Oh} \mathrm{Mn}^{3+}$ would be antiparallel to the tetrahedral $\mathrm{Mn}^{2+}$, and would act to reduce the large peak in dichroism at $640 \mathrm{eV}$ in Fig. 6(b). However, we do not observe such a reduction in the $640 \mathrm{eV}$ peak. While $\mathrm{MnFe}_{2} \mathrm{O}_{4}$ tends to have a small amount of $\mathrm{Oh} \mathrm{Mn}^{3+}$, this contri- bution to the dichroism is overwhelmed by the $\mathrm{Td} \mathrm{Mn}^{2+}$ dichroism. We therefore conclude that in the MCO layer, octahedral $\mathrm{Cr}^{3+}$ is strongly magnetized by the octahedral $\mathrm{Fe}$ in $\mathrm{Fe}_{3} \mathrm{O}_{4}$, and to a smaller degree tetrahedral $\mathrm{Mn}^{2+}$ is also magnetized.

Thus ferromagnetism is strongly induced in octahedral $\mathrm{Cr}^{3+}$ and is accompanied by the stabilization of octahedral $\mathrm{Co}^{2+}$ near the interface in SCF and SFC heterostructures. On the other hand, there is not a significant contribution of octahedral $\mathrm{Mn}^{3+}$ to the induced ferromagnetism in SMF and SFM heterostructures.

\section{CONCLUSION}

At the interfaces of spinel chromite and $\mathrm{Fe}_{3} \mathrm{O}_{4}$ bilayers, we observed induced ferrimagnetic polarization of the $\mathrm{Cr}, \mathrm{Mn}$ and Co lattices with the chromite $\mathrm{Cr}$ and $\mathrm{Fe}_{3} \mathrm{O}_{4}$ octahedral Fe sublattices coupled ferromagnetically. Induced magnetism at the $\mathrm{Fe}_{3} \mathrm{O}_{4} / \mathrm{CCO}$ interface region can persist up to $500 \mathrm{~K}$ with a $3 \mathrm{~nm} \mathrm{CCO}$ layer and up to room temperature with a $6 \mathrm{~nm} \mathrm{CCO}$ layer. A similar ferrimagnetism beyond the bulk Curie temperature was measured in $\mathrm{Fe}_{3} \mathrm{O}_{4} / \mathrm{MCO}$ layers. Chemical and magnetic characterization of the interface indicated that the strong coupling in this isostructural spinel system is due to the stabilization of a spinel phase at the interface not attainable in the bulk, and a proximity-induced magnetism from the adjacent $\mathrm{Fe}_{3} \mathrm{O}_{4}$.

\section{Acknowledgments}

The authors would like to thank Prof. Angelica Stacy for the use of her $\theta-2 \theta$ diffractometer and Dr. Kin Man Yu from the Lawrence Berkeley National Laboratory Materials Science Division for taking RBS spectra. This research was supported by the National Science Foundation (DMR 0604277). JSB was supported by the Office of Naval Research. BBNC and The Advanced Light Source were supported by the Director, Office of Science, Office of Basic Energy Sciences, of the U.S. Department of Energy under Contract No. DE-AC02-05CH11231.
* Electronic address:rajeshch@berkeley.edu

1 W. H. Meiklejohn and C. P. Bean, Physical Review 102, 1413 (1956).

2 S. S. P. Parkin, N. More, and K. P. Roche, Physical Review Letters 64, 2304 (1990).

3 J. Nogués, D. Lederman, T. J. Moran, and I. K. Schuller, Physical Review Letters 76, 4624 (1996).

4 S. Maat, K. Takano, S. S. P. Parkin, and E. E. Fullerton, Physical Review Letters 87, 087202 (2001).

${ }^{5}$ D. T. Margulies, F. T. Parker, M. L. Rudee, F. E. Spada, J. N. Chapman, P. R. Aitchison, and A. E. Berkowitz, Physical Review Letters 79, 5162 (1997).
${ }^{6}$ Y. Ijiri, J. A. Borchers, R. W. Erwin, S. H. Lee, P. J. van der Zaag, and R. M. Wolf, Physical Review Letters 80, 608 (1998).

7 Y. Suzuki, R. B. van Dover, E. M. Gyorgy, J. M. Phillips, and R. J. Felder, Physical Review B 53, 14016 (1996).

8 G. Hu and Y. Suzuki, Physical Review Letters 89, (2002).

${ }^{9}$ G. Hu, R. Chopdekar, and Y. Suzuki, Journal of Applied Physics 93, 7516 (2003).

10 B. B. Nelson-Cheeseman, R. V. Chopdekar, L. M. B. Alldredge, J. S. Bettinger, E. Arenholz, and Y. Suzuki, Physical Review B 76, 220410 (2007).

11 A. T. Young, J. Feng, E. Arenholz, H. A. Padmore, T. Hen- 
derson, S. Marks, E. Hoyer, R. Schlueter, J. B. Kortright, V. Martynov, et al., Nuclear Instruments and Methods in Physics Research Section a-Accelerators Spectrometers Detectors and Associated Equipment 467, 549 (2001).

12 P. Nachimuthu, J. H. Underwood, C. D. Kemp, E. M. Gullikson, D. W. Lindle, D. K. Shuh, and R. C. C. Perera, in Eighth International Conference on Synchrotron Radiation Instrumentation. San Francisco, CA (2004).

13 S. Anders, H. A. Padmore, R. M. Duarte, T. Renner, T. Stammler, A. Scholl, M. R. Scheinfein, J. Stohr, L. Seve, and B. Sinkovic, Review of Scientific Instruments 70, 3973 (1999).

14 R. V. Chopdekar, G. Hu, A. C. Ford, and Y. Suzuki, Journal of Electronic Materials 33, 1254 (2004).

15 B. D. Schrag, A. Anguelouch, S. Ingvarsson, X. Gang, L. Yu, P. L. Trouilloud, A. Gupta, R. A. Wanner, W. J. Gallagher, P. M. Rice, et al., Applied Physics Letters 77, 2373 (2000).

${ }^{16}$ L. F. Kourkoutis, D. A. Muller, Y. Hotta, and H. Y. Hwang, Applied Physics Letters 91, 163101 (2007).

17 N. Keller, A. Das, M. Guyot, M. Porte, and R. Krishnan, Solid State Communications 105, 333 (1998).

18 S. Gota, M. Gautier-Soyer, and M. Sacchi, Phys. Rev. B 62, 4187 (2000).
19 K. Tomiyasu, J. Fukunaga, and H. Suzuki, Phys. Rev. B 70, 214434 (2004).

20 J. Chen, D. J. Huang, A. Tanaka, C. F. Chang, S. C. Chung, W. B. Wu, and C. T. Chen, Physical Review B 69, 085107 (2004).

21 R. A. D. Pattrick, G. Van der Laan, C. M. B. Henderson, P. Kuiper, E. Dudzik, and D. J. Vaughan, European Journal of Mineralogy 14, 1095 (2002).

22 E. Arenholz, G. van der Laan, R. V. Chopdekar, and Y. Suzuki, Physical Review B 74, 094407 (2006).

23 N. Menyuk, K. Dwight, and A. Wold, J. Phys. France 25, 528 (1964).

24 H. S. C. O'Neill and A. Navrotsky, American Mineralogist 68, 181 (1983).

25 B. Lavina, G. Salviulo, and A. Della Giusta, Physics and Chemistry of Minerals 29, 10 (2002).

${ }^{26}$ H. Mohan, I. A. Shaikh, and R. G. Kulkarni, Physica B: Condensed Matter 217, 292 (1996).

27 M. H. Francombe, Journal of Physics and Chemistry of Solids 3, 37 (1957).

28 Y. G. Saksonov and V. A. Somenkov, Fizika Metallov i Metallovedenie 18, 853 (1964). 